\title{
Electronic Risk Assessment System as an Appropriate Tool for the Prevention of Cancer: a Qualitative Study
}

\author{
Amir hossein Javan Amoli ${ }^{1}$, Elham Maserat ${ }^{2 *}$, Reza Safdari ${ }^{2}$, Mohammad Reza \\ Zali $^{3}$
}

\begin{abstract}
Background: Decision making modalities for screening for many cancer conditions and different stages have become increasingly complex. Computer-based risk assessment systems facilitate scheduling and decision making and support the delivery of cancer screening services. The aim of this article was to survey electronic risk assessment system as an appropriate tool for the prevention of cancer. Materials and Methods: A qualitative design was used involving 21 face-to-face interviews. Interviewing involved asking questions and getting answers from exclusive managers of cancer screening. Of the participants 6 were female and 15 were male, and ages ranged from 32 to 78 years. The study was based on a grounded theory approach and the tool was a semistructured interview. Results: Researchers studied 5 dimensions, comprising electronic guideline standards of colorectal cancer screening, work flow of clinical and genetic activities, pathways of colorectal cancer screening and functionality of computer based guidelines and barriers. Electronic guideline standards of colorectal cancer screening were described in the $\mathbf{3} 3$ categories of content standard, telecommunications and technical standards and nomenclature and classification standards. According to the participations' views, workflow and genetic pathways of colorectal cancer screening were identified. Conclusions: The study demonstrated an effective role of computer-guided consultation for screening management. Electronic based systems facilitate real-time decision making during a clinical interaction. Electronic pathways have been applied for clinical and genetic decision support, workflow management, update recommendation and resource estimates. A suitable technical and clinical infrastructure is an integral part of clinical practice guidline of screening. As a conclusion, it is recommended to consider the necessity of architecture assessment and also integration standards.
\end{abstract}

Keywords: Electronic risk assessment system - prevention - cancer screening - colorectal

Asian Pac J Cancer Prev, 16 (18), 8595-8598

\section{Introduction}

Colorectal (CRC) is one of the most common cancers in worldwide (Safaee et al., 2008). But it is one of the most preventable cancers (Atkinson et al., 2008). More studies have proved that colorectal cancer incidence and mortality are reduced with regular screening (Gooran et al., 2010). Colorectal Cancer control program of Shaheed Beheshti research institute for gastroenterology and liver disease of Iran (RIGLD) supports population-based screening efforts in many provinces. The RIGLD's goal is to increase colorectal cancer screening rates among men and women aged 50 years. But decision strategies for screening have become increasingly complex in recent decades. Screening pathway and guidelines recommended more and new options for cancer management (Masahito et al., 2013). The use of computer technologies facilitate decision making and improve efficiency of screening process (Maserat et al., 2012). Decisions of computer base system could be a suitable tool to help patients understand their risk of developing a particular cancer, the screening approach, survival of cancer, recommended screening time intervals, preferences for a special option, assessment of patients' needs, treatment option and probabilities of outcome (Bouaud et al., 2014; Dekker et al., 2014; Eccher et al., 2014 Steele et al., 2014; Yilmaz et al., 2015). Computer based guideline and electronic knowledge based system support of medical decisionmaking, selection of the accurate diagnosis and suitable treatment plan (Tomaszewski et al., 2012). Electronic clinical practice guidelines (CPGs) enhance the quality of screening and reduce health care costs. Computerized CPGs provide patient specific advices when and where needed for health care professionals (Peleg et al., 2013). Colorectal cancer screening management, especially for those with a genetic predisposition depends on patient specific advices. These accurate reports improve quality of care and clear communication among health care providers

${ }^{1}$ Islamic Azad University, ${ }^{2}$ School of Allied Medical Sciences, Tehran University of Medical Sciences, ${ }^{3}$ Research Center of Gastroenterology and Liver Diseases, Shahid Beheshti University of Medical Science, Tehran, Iran *For correspondence: elhammaserat@gmail.com 
(Maserat et al., 2009). A number of studies have shown positive options for multidimensional guidelines such as ambulatory services or reminder systems for preventive care services (Heselmans et al., 2009). However computerguided consultation improves real time management (Angus et al., 2012). An accurate computerized guideline need to integrate with workflow of screening (Shiffman et al., 1999). Standard document formats is integral part for combining of computerized guideline and workflow of screening (Lobach et al., 1994). A vast amount of information has been produced in screening plan of RIGLD. Managing of this information needs to analyzing approach such as multidimensional guideline. According to studies, computer based guideline facilitates information management process and recommends patient-specific advice. The aim of this article is to survey computer based guideline as an effective approach for the management of colorectal cancer screening.

\section{Materials and Methods}

A qualitative design was used involving 21 face-toface interviews with health care managers. 6 participants were female and 15 were male, and ages ranged from 32 to 78 years. Interview duration ranged between 15 and 30 minutes. Interviewing involves asking questions and getting answers from participants. This review described computer based guideline as effective approach for managing of colorectal cancer screening.

The study was based on the grounded theory approach (Glaser et al., 1967). Study tool was semi-structured interview. 21 managers and stakeholders 'perspectives were reviewed. This sample provided sufficient numbers to ensure exploration of the fields, and data saturation was reached by the final interviews. Participants were informed about colorectal cancer screening program and computerized multidimensional guidelines. Also participants have had experience on cancer screening field. Managers were asked about electronic guideline standards of colorectal cancer screening, work flow of clinical and genetic activities, pathways of colorectal cancer screening and functionality of computer based guidelines and barriers. All interviews were fully transcribed and coded and analyzed by two researchers. Participants were recruited from research centers and a hospital with cancer screening activities. The researcher explained the purpose of the study and confidentially of information for participation. Also researcher asked for consent to audio-record the interviews. Data was gathered by two researchers between April and Jun 2015. Finally coded data was organized.

\section{Results}

Researchers studied 5 dimensions, including electronic guideline standards of colorectal cancer screening, work flow of clinical and genetic activities, pathways of colorectal cancer screening and functionality of computer based guidelines and barriers.

\section{screening}

Electronic guideline Standards of colorectal cancer screening were described in 3 categories including content standard, telecommunications and technical standards and nomenclature and classification standard.

Standard documentation systems of cancer screening facilitate quality improvement of control program according to participations' views. Content standard refer to core data set of guidelines. Content standard was contained colonoscopy, pathology, surgery, genetics and pedigree information. Content standard of colonoscopy includes demographic and medical history data set, administrative data, study data set, finding data set and follow up data set. Content standard of pathology classified to macroscopic and microscopic features. Also content standard of surgery was classified to Specimen and Tumor specification. Genetic information was included IHC (ImmunoHistoChemistry) and MSI (Microsatellite instability) test information, APC (Adenomatous polyposis coli) and MMR (Mismatch Repair Genes) information and HNPCC (Hereditary non-polyposis colorectal cancer) and FAP (Familial adenomatous polyposis;) results. Pedigree information play significance role in planning of colorectal cancer screening especially for indentifying high risk population by perspectives.

Nomenclature and classification standards facilitate electronic transmission of results to laboratory, hospital, physician, third party payers, and other users of data. The LOINC (Logical Observation Identifier Names and Codes) and SNOMED (Systematized Nomenclature of Medicine Clinical Terms) were selected as nomenclature standards by managers and ICD-O (International Classification of Diseases for Oncology ) as classification standard was selected. Nomenclature and classification standards allow a consistent way to index, store, retrieve, and aggregate clinical data across health care professionals and sites of care. These standards provide comprehensive and precise clinical content for documentation and reporting.

HL 7 and DICOM were introduced as telecommunications \& technical standard by studied experts. XML and RDF are examples for standard syntactic frameworks. NLP was introduced as mapping tool that match with UMLS (Unified Medical Language System) metathesaurus.

\section{Work flow of clinical and genetic activities}

Table 1 was illustrated work flow of clinical and genetic activities by participations' views. Functions of colorectal cancer screening contain research, education, clinical activities and health information management and other activities. Education department contain contentious education to patients and high risk people. Educational tools were website, forum, newspaper, educational package, workshops and conference. Clinical and genet activities include.

i) Colonoscopy and other related activities. Target population is fist degree relatives of proband. ii) Pathology examination and CRC block analyzing. iii) Genetic testing: Surveying IHC, MSI and detection of hereditary non-polyposis colorectal cancer (HNPCC) and (familial adenomatous polyposis) FAP. One of positive view of 
Table 1. Work flow of Clinical and Genetic Activities of Colorectal Cancer Screening by Participations' Views

\begin{tabular}{ll}
\hline Clinical Work Flow & Genetic Work Flow \\
\hline 1) Patient finding & 1) Genetic counseling \& drawing the pedigrees \\
2) Educational Counseling & 2) Collecting peripheral blood \& one paraffin embedded block DNA extraction \\
3) Medical documentation and drawing pedigree & 3) Immunohistochemistry ( IHC ) \\
4) Blood sampling and taking pathology block & 4) Microsatellite Instability ( MSI ) \\
5) Genetic and pathology examination & Genetic testing including PCR, gel \\
6) Identify high risk population &
\end{tabular}

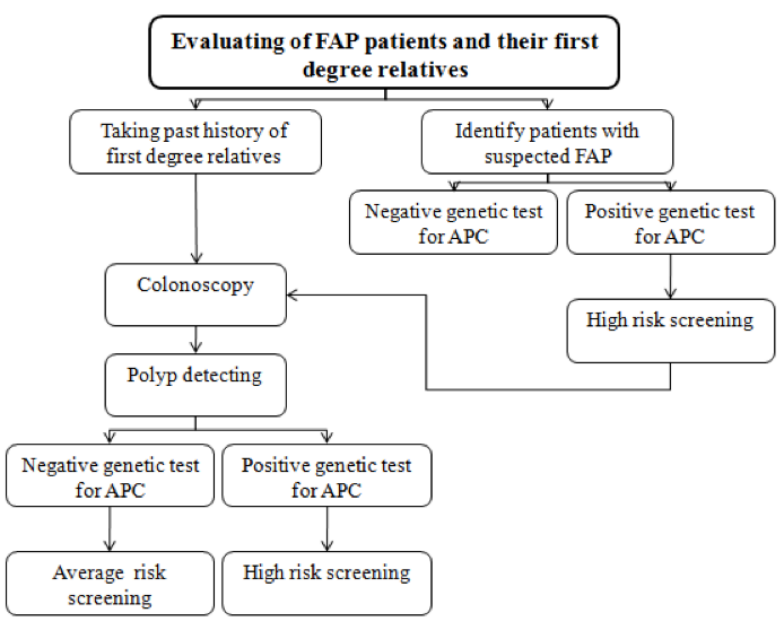

Figure 1. Familial Adenomatous Polyposis Pathway by Participations' Views

plan is special attention to genetics because this issue has impacted on high risk populations diagnostics.

Pathways of colorectal cancer screening

FAP and HNPCC are two syndromes of colorectal cancer predisposition. Figure 1 and 2 were presented Genetic pathway by participations' views.

\section{Functionality of computer based guidelines}

According to participations' views, computer based guidelines of screening comprises seven functions of information management of screening, defined as follows:

Registration: The collection, storage of administrative and demographic data related to uniquely identify the patient, health care provider(s), and encounter.

Recommendation based on clinical guidelines: Guideline-specified functions that should occur under specific clinical circumstances for example: identify high risk population (FAP, HNPCC), identify average and at risk population, planning colonoscopy times, early detection, recurrence prediction, burden prediction and survival prediction.

Managerial decision making: the provision of background information for resource requirements estimate, time management of personnel, measures of quality or cost and finally quality improvement of process.

Presentation: the creation of effective output from integrated genetics, pathology, colonoscopy and surgery information.

Analyzing and disseminating: the manipulation of numeric or nominal data, or both, to derive required information (e.g., observations, pedigree assessments, and interventions related to clinical care).

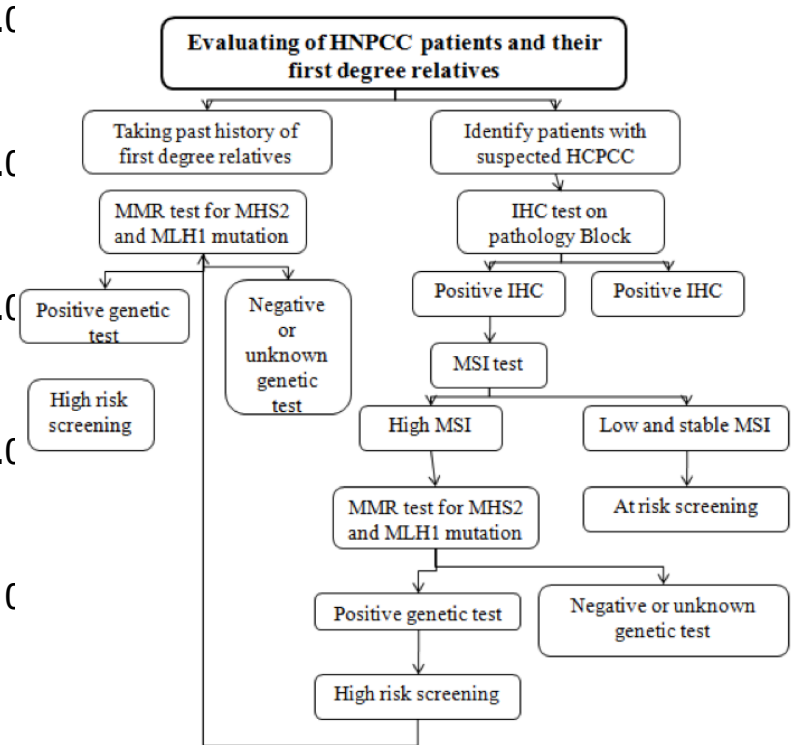

Figure 2. Hereditary Non-polyposis Colorectal Cancer Pathway by Participations' Views

Aggregation: the derivation of population-based cancer screening information from individual patient data.

Communication: The interaction between the clinicians and other providers of cancer screening plan by electronic messages.

\section{Barriers of computer based guidelines implementing}

According to participations' views, transformation of screening guideline to computer program can be difficult from a technical aspect. Adoption of terminologies and data exchange standard was a difficult process. Also prioritization for recommendations and categorizing of screening protocol was complex. User interface for the presentation of reminders and recommendation of pathway was not user friendly. Other main barriers were inappropriate architecture and evaluation methods.

\section{Discussion}

Standard of clinical practice guidelines for driving care management are becoming increasingly important (Lobach et al., 1994). The use of the standard model for representation of sharable computer guidelines is integral part of guideline implementing. The Guideline Interchange Format (GLIF) is a one of the standard model (Boxwala et al., 2004). This study was surveyed content standard, telecommunications \& technical standard, nomenclature and classification standard. According to participations' views, adoption of terminologies and other standards was 
a difficult process. We need comprehensive standards for the sharing of screening content.

The more studies demonstrate the importance of evaluating workflow and information flow in health information technology design and implementation (Unertl et al., 2009). The study demonstrates work flow of clinical and genetic activities for colorectal cancer screening participations' views. Pathways of colorectal cancer screening identified gaps between the existing guidelines functionality and the needs of providers of screening program. The authors demonstrated two pathways of screening including FAP and HNPCC. Appropriate cancer management requires an understanding of several features that help to early detection. CPG direct and identify features (R. Collado et al., 2014). Functions of CPG support of features such as differentiation, behavior and histology of tumors. Computer-guided consultations are practicable in chronic disease (Angus et al., 2012). Recommendation based on clinical guidelines help to risk assessment and scheduling of colonoscopy times.

In Conclusions: Study was demonstrated the effective role of computer-guided consultation for screening management. Electronic risk assessment systems facilitate real-time decision making during a clinical interaction. Electronic pathways have been applied for clinical and genetic decision support, workflow management, update recommendation and resource estimates. One of the key aspects to achieve comprehensive computer based guideline of colorectal cancer screening are the usage of common or interoperable content (colonoscopy, pathology and genetic terminology), data exchange standards and technical integration. Also a suitable technical and clinical infrastructure is integral part of CPG of screening. Screening guidelines need to be integrated with the care flow for providing suitable patient-specific advice. After more than a decade of development of numerous computerized systems, studies on the most effective implementation of Electronic risk assessment systems is still lacking. Considering to findings of this study is useful for optimal implementing of Electronic risk assessment system of colorectal cancer screening. As a conclusion, it is recommended to consider the necessity of architecture assessment and also integration standards

\section{Acknowledgements}

This study was supported by the Research Institute of Gastroenterology and Liver Diseases, Shahid Beheshti University of Medical Science. We thank Fatemeh Ghafarnejad, Mohammad Amin Pourhosseingholi for providing counseling.

\section{References}

Angus RM, Thompson EB, Davies L, et al (2012). Feasibility and impact of a computer-guided consultation on guidelinebased management of COPD in general practice. Prim Care Respir J, 21, 425-30.

Atkinson W (2008). Study aims to improve colorectal cancer screening participation. AHIP Cover, 42, 46-8.

Bouaud J, Blaszka-Jaulerry B, Zelek L, et al (2014). Health information technology: use it well, or don't! Findings from the use of a decision support system for breast cancer management. AMIA Annu Symp Proc, 14, 315-24.

Boxwala AA, Peleg M, Tu S, et al (2004). GLIF3: a representation format for sharable computer-interpretable clinical practice guidelines. J Biomed Inform, 37, 147-61.

Collado Martín R, García Palomo A, de la Cruz Merino L, Borrega García P, Barón Duarte FJ (2014). Clinical guideline SEOM: cancer of unknown primary site. Clin Translational Oncology, 16, 1091-7.

Dekker A, Vinod S, Holloway L, et al (2014). Rapid learning in practice: a lung cancer survival decision support system in routine patient care data, Radiother Oncol, 113, 47-53.

Eccher C, Seyfang A, Ferro A (2014).Implementation and evaluation of an Asbru-based decision support system for adjuvant treatment in breast cancer. Comput Methods Programs Biomed, 117, 308-21.

Eriksson H, Tu SW, Musen M. Semantic clinical guideline documents (2005). AMIA Annu Symp Proc, 236-40.

Glaser B, Strauss A (1967). The Discovery of Grounded Theory. Chicago, IL: Aldine.

Heselmans A, Van de Velde S, Donceel P, Aertgeerts B, Ramaekers D (2009). Effectiveness of electronic guidelinebased implementation systems in ambulatory care settings - a systematic review. Implement Sci, $\mathbf{3 0}$.

Lobach DF, Hammond WE (1994). Development and evaluation of a computer-assisted management protocol (CAMP): improved compliance with care guidelines for diabetes mellitus. Proc Annu Symp Comput Appl Med Care,787-91.

Jimbo M, Rana GK, Hawley S, et al (2013). What is lacking in current decision aids on cancer screening? CA: A Cancer Journal for Clinicians, 63, 193-214.

Maserat E, Reza S, Elnaz M, Mohamad Reza Zali (2012). Endoscopic electronic record: A new approach for improving management of colorectal cancer prevention. World $J$ Gastrointest Oncol, 4, 76-81.

Maserat Elham, Reza Fatemi, Mohamad Reza Zali (2009). New perspective for integrated information management in national colorectal cancer screening in Iran. Asian Pac J Cancer Prev, 10.

Navid G, Shima A, Elham M, Masoume S, Reza FS (2010). The role of information systems in cancer prevention. Gastroenterology and Hepatology From Bed to Bench, 3, 164-168.

Peleg M (2013). Computer-interpretable clinical guidelines: a methodological review. J Biomed Inform, 46, 744-63.

Safaee A, Moghimi-Dehkordi B, Fatemi SR, et al (2008). Colorectal cancer in Iran. Asian Pac J Cancer Prev, 9, 123-6.

Shiffman RN1, Brandt CA, Liaw Y, Corb GJ (1999). A design model for computer-based guideline implementation based on information management services. J Am Med Inform Assoc, 6, 99-103.

Steele SR, Bilchik A, Johnson EK, et al (2014). Time-dependent estimates of recurrence and survival in colon cancer: clinical decision support system tool development for adjuvant therapy and oncological outcome assessment. Am Surg, 80, 441-53.

Tomaszewski W (2012). Computer-based medical decision support system based on guidelines, clinical pathways and decision nodes. Acta Bioeng Biomech, 14, 107-16.

Unertl KM, Weinger MB, Johnson KB, Lorenzi NM (2009). Describing and modeling workflow and information flow in chronic disease care. J Am Med Inform Assoc, 16, 826-36.

Yilmaz AA, Ozdemir L (2015). Development and implementation of the clinical decision support system for patients with cancer and nurses' experiences regarding the system. Int $J$ Nurs Knowl. 